\title{
Family History of Coronary Artery Disease
}

National Cancer Institute

\section{Source}

National Cancer Institute. Family History of Coronary Artery Disease. NCI Thesaurus. Code C80398.

History of a first-degree relative (less than 55 years for male relatives or less than 65 years for female relatives) having had any of the following: coronary artery disease, myocardial infarction, coronary artery bypass graft surgery, percutaneous coronary intervention, or sudden cardiac death without obvious cause. 